\title{
COMPARAÇÃO ENTRE AS REDES NEURAIS ADALINE E PERCEPTRON UTILIZANDO O CONJUNTO DE DADOS IRIS
}

\author{
Comparison between the neural networks Adaline and Perceptron using the \\ IRIS database
}

\author{
Allana dos Santos Campos e César Alberto Bravo Pariente \\ Universidade Estadual de Santa Cruz - UESC, BA \\ E-mail: allanacampos50@gmail.com, cabpariente@uesc.br
}

\begin{abstract}
RESUMO - Inicialmente as redes neurais foram desenvolvidas com o objetivo de criar um sistema computacional que modele o funcionamento do cérebro humano, porém as mesmas passaram a ser utilizadas para resolver tarefas específicas. Adaline e Perceptron são duas redes neurais que calculam uma função de entrada utilizando um conjunto de pesos adaptativos e um bias e, apesar de suas similaridades, é conhecido que o Adaline converge para um resultado mais rapidamente que o Perceptron. Este trabalho foi elaborado como um exercício didático, afim de apresentar como se obtém tais conclusões, utilizando como dados para classificação e treinamento o conjunto de dados IRIS. Ao longo do trabalho utilizou-se as linguagens de programação Processing, para elaboração das redes neurais, e Python para apresentação visual dos resultados. Os resultados encontrados apresentam o desempenho superior da rede neural Adaline sobre o Perceptron, evidenciando as classes do conjunto de dados que podem ser separadas linearmente e as que não podem, a métrica utilizada para avaliar o desempenho entre as redes neurais é definida pela porcentagem de acertos nas classificações dos dados. O Adaline apresentou melhor desempenho na classificação para comprimento e largura da pétala entre as classes Iris-setosa e Iris-virginica dentre todas as demais classificações.
\end{abstract}

Palavras-chave: Redes Neurais; Adaline; Perceptron.

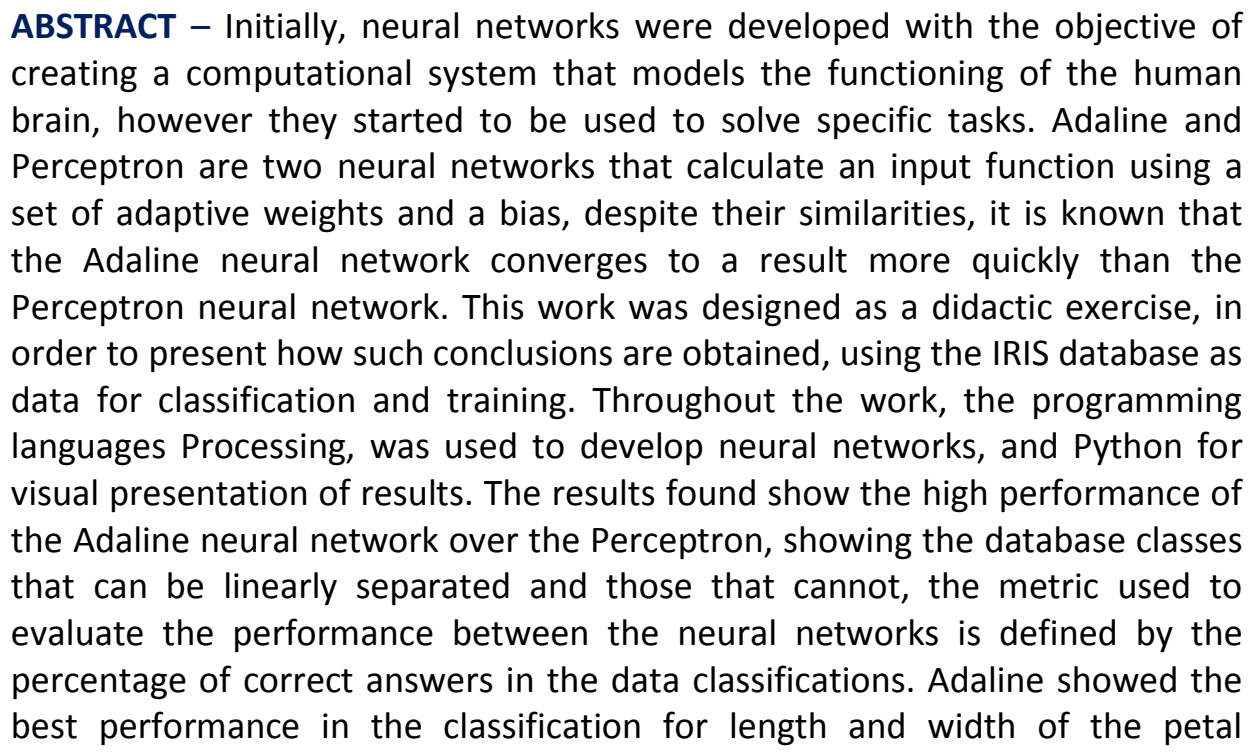


between the Iris-setosa and Iris-virginica classes among all the other classifications.

Keywords: Neural Network; Adaline; Perceptron.

\section{INTRODUÇÃO}

Redes neurais artificias são sistemas que possuem vários neurônios artificiais interconectados com o propósito de simular o processo de aprendizagem do cérebro humano. Ao implementar uma rede neural artificial, ela se torna capaz de reconhecer padrões e correlações, agrupar e classificar dados, por conta de tais utilidades as redes neurais são principalmente utilizadas para criar sistemas de inteligência artificial.

Uma das redes neurais mais conhecidas é o Perceptron, por possuir uma arquitetura simples é considerado um bom modelo introdutório. Foi desenvolvido nas décadas de 1950 e 1960 pelo cientista Frank Rosenblatt, inspirado em trabalhos anteriores de Warren McCulloch e Walter Pitts [Data Science Academy, 2019].

Um perceptron é um modelo matemático que a partir de vários valores como entrada devolve uma saída binária. Para simular o processo de aprendizagem o perceptron possui pesos sinápticos para cada entrada que são atualizados até que resultem em um valor desejado para a saída.

A rede neural Adaline, do inglês: Adaptive Linear Neuron, foi proposta por Widrow e Hoff, 1960 [Moreira, 2018], e possui a mesma estrutura que o Perceptron, se diferenciando apenas no algoritmo de treinamento conhecido como Regra Delta e possuindo saídas bipolares.

A Regra Delta foi desenvolvida devido à incapacidade do algoritmo de treinamento do Perceptron em gerar pesos que classifiquem dados não linearmente separáveis. $O$ algoritmo para o Adaline realiza iterações locais para obter o ponto mínimo da função de erro e, assim, fixa os valores dos pesos quando o mínimo foi encontrado [Moreira, 2018].

Com intuito de comparar as duas redes neurais, Adaline e Perceptron, este trabalho foi desenvolvido utilizando o conjunto de dados IRIS, que pode ser encontrado em [Dua e Graff, 2019], para treinamento e classificação das redes neurais.

Em [Fisher, 1936] o estatístico e biólogo britânico Ronald Fisher introduziu o conjunto de dados IRIS, que possui 150 amostras de três espécies de Iris, Iris-setosa, Iris-versicolor e Irisvirginica, cada amostra possui quatro características, o comprimento e largura da sépala e o comprimento e largura da pétala.

\section{MATERIAIS E MÉTODOS}

\subsection{Materiais}

- $\quad$ Computador Intel ${ }^{\circledR}$ Core $^{\mathrm{TM}}$ i7 $7500 \mathrm{CPU}$ @ 2.70GHz, 2.90GHz, 8.00GB, 64 bits, Windows 10;

- $\quad$ Python 3.7.4;

- $\quad$ Processing 3.5.4;

- $\quad$ Conjunto de Dados Iris;

\subsection{Métodos}

Para cada rede neural foram implementados dois algoritmos, que podem ser encontrados em [Autor, 2020], utilizando a linguagem de programação Processing. Os algoritmos foram baseados nos pseudocódigos encontrados no livro de Danilo G. Spatti, Ivan N. da Silva e Rogério A. Flauzino, "Redes Neurais Artificiais Para Engenharias e Ciências Aplicadas" [Silva, 2010], a seguir:

Considere $w$ como o vetor com os valores dos pesos sinápticos. A função 'sinal(u)' implementada foi: se $u \leq-1$ então $y=0$, caso contrário $\mathrm{y}=1$.

Figura 1. Pseudocódigo para treinamento da rede neural Perceptron

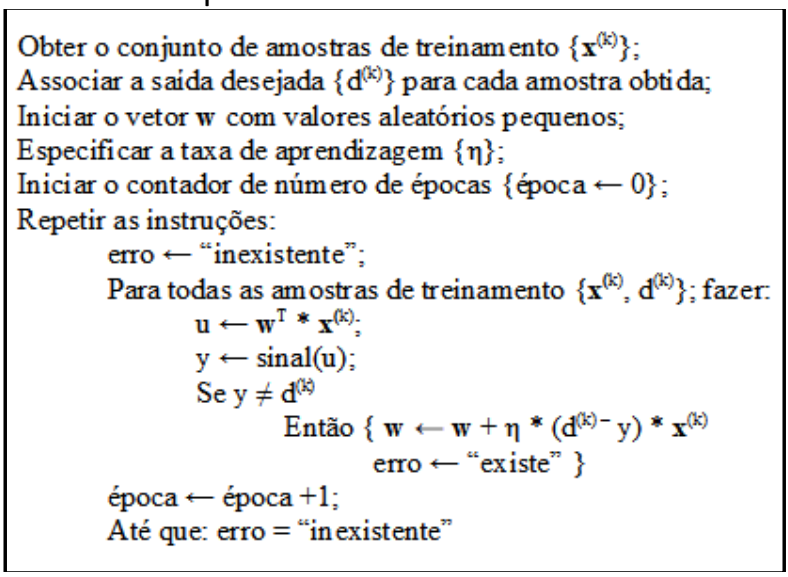

Fonte: (Silva, 2010) 
Como a função 'sinal(u)' utilizada não devolve o valor '-1' então na implementação da classificação a amostra $\mathrm{x}$ pertence a Classe $\mathrm{A}$ se $\mathrm{y}$ $=0$.

Figura 2. Pseudocódigo para classificação da rede neural Perceptron

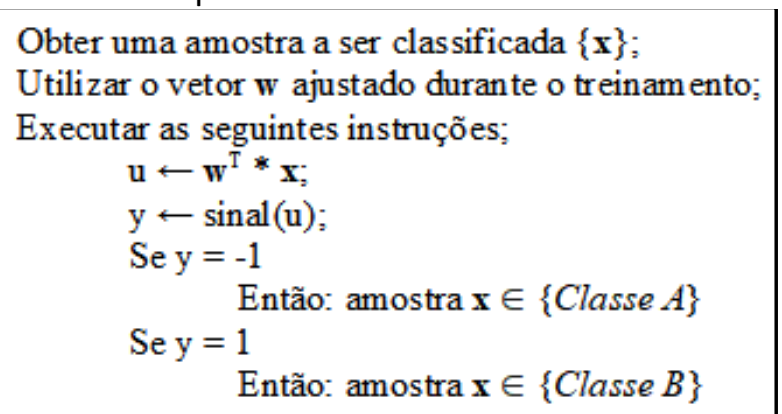

Fonte: (Silva, 2010)

Para o pseudocódigo da Adaline, considere que $\mathrm{w}$ é o vetor com os valores dos pesos sinápticos, assim como no algoritmo de treinamento do Perceptron.

Figura 3. Pseudocódigo para treinamento da rede neural Adaline

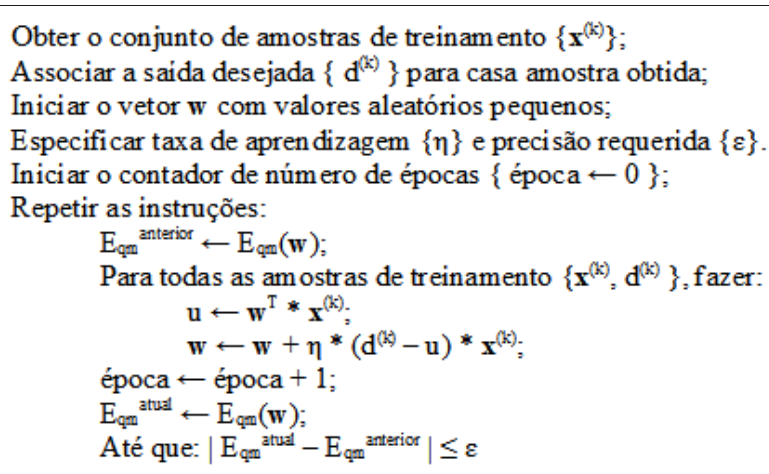

Fonte: (Silva, 2010)

A função do erro quadrático médio $(E q m(w))$ encontrada no algoritmo para o treinamento foi baseada no pseudocódigo a seguir:

Figura 4. Pseudocódigo para função do erro quadrático médio da rede neural Adaline

\footnotetext{
Obter a quantidade de padrões de treinamento $\{\mathrm{p}\}$;

Iniciar a variável $\mathrm{E}_{\mathrm{qm}}$ com valor zero $\left\{\mathrm{E}_{\mathrm{qm}} \leftarrow 0\right\}$;

Para todas as amostras de treinamento $\left\{\mathbf{x}^{(k)}, d^{(k)}\right\}$, fazer: $\mathrm{u} \leftarrow \mathbf{w}^{\mathrm{T}} * \mathbf{x}^{(\mathrm{k})}$;$$
\mathrm{E}_{\mathrm{qm}} \leftarrow \mathrm{E}_{\mathrm{qm}}+\left(\mathrm{d}^{(\mathrm{k}) * \mathrm{u})^{2}}\right.
$$

$\mathrm{E}_{\mathrm{qm}} \leftarrow \mathrm{E}_{\mathrm{qm}} / \mathrm{p}$;
}

Fonte: (Silva, 2010)
Figura 5. Pseudocódigo para classificação da rede neural Adaline

Obter uma amostra a ser classificada $\{\mathbf{x}\}$;

Utilizar o vetor $\mathbf{w}$ ajustado durante o treinamento;

Executar as seguintes instruções:

$$
\begin{aligned}
& \mathrm{u} \leftarrow \mathbf{w}^{\mathrm{T} *} \mathbf{x} ; \\
& \mathrm{y} \leftarrow \operatorname{sinal}(\mathrm{u}) ; \\
& \text { Se } \mathrm{y}=-1 \\
& \quad \text { Então: amostra } \mathbf{x} \in\{\text { Classe } A\} \\
& \text { Se y }=1 \\
& \quad \text { Então: amostra } \mathbf{x} \in\{\text { Classe } B\}
\end{aligned}
$$

Fonte: (Silva, 2010)

Foram elaborados três arquivos a partir do conjunto de dados IRIS, um arquivo com todos os valores referentes as classes Iris-setosa e Irisversicolor (setvers.txt), um arquivo com todos os valores referentes as classes Iris-setosa e Irisvirginica (setvirg.txt) e um arquivo com todos os valores referentes as classes Iris-versicolor e Irisvirginica (virgvers.txt).

Para gerar o conjunto de dados para o treinamento e classificação das redes neurais foi elaborado um programa, 'geradado', utilizando a linguagem de programação Processing, que contabiliza a quantidade de linhas dos arquivos elaborados pelo conjunto de dados IRIS, sorteia um número entre 0 e a quantidade de linhas, copia a linha representada pelo número no arquivo de saída e salva o número sorteado em um vetor para comparar com os próximos sorteios para não repetir a linha.

Como os arquivos de entrada, setvers.txt, setvirg.txt e virgvers.txt, possuem a metade superior de linhas com amostras de uma classe e a metade inferior de linhas com amostras de outra classe, primeiramente o programa sorteia metade das linhas desejadas para o arquivo de saída nas linhas referentes a classe apresentada no início do arquivo e o restante nas linhas referentes a classe apresentada do final do arquivo de entrada. Tal arquivo de saída servirá como arquivo de treinamento. As linhas que não foram sorteadas são copiadas a outro arquivo de saída que servirá como arquivo de classificação.

Para ajudar na comparação e avaliação dos resultados foi elaborado um gráfico $4 X 4$ utilizando a linguagem de programação python, apresentando as combinações das características das amostras encontradas do conjunto de dados. Inicialmente o arquivo com as amostras teve o formato alterado para CSV para que fosse lido com a função 'read_csv' da biblioteca pandas [Python, 2020], as colunas de cada gráfico foram 
definidas como as características de cada amostra e com a função 'pairplot' da biblioteca seaborn [Waskom, 2020] foi gerado o gráfico.

Também foram elaborados gráficos utilizando a biblioteca matplotlib [Hunter, 2007] da linguagem de programação python, utilizando os valores dos pesos resultantes do treinamento das redes neurais e a função 'pyplot.plot' para a geração de uma reta, $w_{1}{ }^{*} x+w_{2}{ }^{*} y+\theta=0$, onde $w_{1}$ e $W_{2}$ representam os valores dos pesos sinápticos e $\theta$ o valor do bias, e, os valores resultantes da classificação e a função 'pyplot.scatter' para a geração de pontos no plano.

\section{DESENVOLVIMENTO}

Os algoritmos para o Adaline e Perceptron apresentam uma única saída como resposta, de modo que classificam apenas duas classes. Como o conjunto de dados IRIS apresenta três classes distintas, Iris-setosa, Iris-versicolor e Iris-virginica, foram definidas três combinações entre as classes para serem classificadas, Irissetosa e Iris-versicolor, Iris-setosa e Iris-virginica, Iris-versicolor e Iris-virginica.

O conjunto de dados IRIS contém 150 amostras classificadas, 50 amostras de cada classe, para o treinamento das redes neurais sete conjuntos de treinamento foram elaborados contendo de 20 a 80 amostras, com incrementos de 10 , que foram escolhidas aleatoriamente do conjunto de dados. $O$ restante das amostras de cada conjunto de dados formaram os sete conjuntos de classificação.

Para o treinamento e classificação das redes neurais, cada conjunto de entrada possui quatro valores que representam o comprimento e largura da sépala e o comprimento e largura da pétala. Com o intuito de visualizar os resultados da classificação no plano, os conjuntos de entrada foram divididos, de modo que sejam treinados e classificados duas características por vez.

Para a saída desejada para cada conjunto de entrada foram utilizados os números 1 e 0 para representar as classes no Perceptron e os números 1 e -1 para representar as classes no Adaline, de forma que na classificação entre Irissetosa e Iris-versicolor o 1 representa a Irissetosa e o 0 a Iris-versicolor para o Perceptron e para o Adaline o-1 representa a Iris-versicolor; na classificação entre Iris-setosa e Iris-virginica são os mesmos valores para Iris-setosa e a Irisvirginica é representada pelos mesmos valores da Iris-versicolor, na classificação entre Iris- versicolor e Iris-virginica o valor 1 representa a Iris-virginica e os valores 0 e -1 a Iris-versicolor.

As redes neurais foram treinadas para cada conjunto de dados e com os pesos obtidos no treinamento foram geradas as classificações das amostras. Foram geradas tabelas com o percentual de erros e acertos de cada resultado. Também foram gerados gráficos com os planos de corte e os pontos de cada classificação para comparação das redes neurais.

\section{RESULTADOS}

Em cada classificação utilizou-se 7 conjuntos de treinamento, nas tabelas a seguir estão o melhor desempenho, com maior porcentagem de acertos, e o pior desempenho, com menor porcentagem de acertos, encontrados em cada classificação. Nos casos em que na mesma classificação foi encontrado o mesmo desempenho para diferentes conjuntos de treinamento sendo ele o pior ou melhor valor encontrado, o valor é adicionado nas tabelas. $\mathrm{O}(\mathrm{s})$ melhor(es) desempenho(s) ocupa(m) o início da tabela, enquanto o(s) pior(es) desempenho(s) ocupa $(m)$ o final da tabela

\subsection{Perceptron}

Tabela 1. Utilizando rede neural Perceptron, classificação para comprimento e largura da sépala das classes Iris-setosa e Iris-versicolor

\begin{tabular}{cccc}
\hline $\begin{array}{c}\text { Conjunto de } \\
\text { Treinamento } \\
\text { (\%) }\end{array}$ & $\begin{array}{c}\text { Acertos } \\
\text { (\%) }\end{array}$ & $\begin{array}{c}\text { Falsos } \\
\text { Setosa (\%) }\end{array}$ & $\begin{array}{c}\text { Falsos } \\
\text { Versicolor } \\
\text { (\%) }\end{array}$ \\
\hline 30.00 & 100.00 & 0.00 & 0.00 \\
70.00 & 100.00 & 0.00 & 0.00 \\
40.00 & 90.00 & 10.00 & 0.00 \\
50.00 & 90.00 & 10.00 & 0.00 \\
\hline
\end{tabular}

Fonte: (Autor, 2020).

Tabela 2. Utilizando rede neural Perceptron, classificação para comprimento e largura da pétala das classes Iris-setosa e Iris-versicolor

\begin{tabular}{cccc}
$\begin{array}{c}\text { Conjunto de } \\
\text { Treinamento } \\
\text { (\%) }\end{array}$ & $\begin{array}{c}\text { Acertos } \\
\text { (\%) }\end{array}$ & $\begin{array}{c}\text { Falsos } \\
\text { Setosa (\%) }\end{array}$ & $\begin{array}{c}\text { Falsos } \\
\text { Versicolor } \\
\text { (\%) }\end{array}$ \\
\hline 20.00 & 100.00 & 0.00 & 0.00 \\
60.00 & 100.00 & 0.00 & 0.00 \\
70.00 & 100.00 & 0.00 & 0.00 \\
80.00 & 95.00 & 5.00 & 0.00 \\
\hline
\end{tabular}

Fonte: (Autor, 2020). 
Tabela 3. Utilizando rede neural Perceptron, classificação para comprimento e largura da sépala das classes Iris-setosa e Iris-virgínica

\begin{tabular}{cccc}
\hline $\begin{array}{c}\text { Conjunto de } \\
\text { Treinamento } \\
\text { (\%) }\end{array}$ & $\begin{array}{c}\text { Acertos } \\
\text { (\%) }\end{array}$ & $\begin{array}{c}\text { Falsos } \\
\text { Setosa (\%) }\end{array}$ & $\begin{array}{c}\text { Falsos } \\
\text { Virginica } \\
\text { (\%) }\end{array}$ \\
\hline 70.00 & 100.00 & 0.00 & 0.00 \\
80.00 & 95.00 & 5.00 & 0.00 \\
\hline
\end{tabular}

Fonte: (Autor, 2020).

Tabela 4. Utilizando rede neural Perceptron, classificação para comprimento e largura da pétala das classes Iris-setosa e Iris-virginica

\begin{tabular}{cccc}
\hline $\begin{array}{c}\text { Conjunto de } \\
\text { Treinamento } \\
\text { (\%) }\end{array}$ & $\begin{array}{c}\text { Acertos } \\
\text { (\%) }\end{array}$ & $\begin{array}{c}\text { Falsos } \\
\text { Setosa (\%) }\end{array}$ & $\begin{array}{c}\text { Falsos } \\
\text { Virginica } \\
\text { (\%) }\end{array}$ \\
\hline 40.00 & 100.00 & 0.00 & 0.00 \\
70.00 & 100.00 & 0.00 & 0.00 \\
80.00 & 100.00 & 0.00 & 0.00 \\
20.00 & 90.00 & 2.50 & 7.50 \\
\hline
\end{tabular}

Fonte: (Autor, 2020).

Tabela 5. Utilizando rede neural Perceptron, classificação para comprimento e largura da sépala das classes Iris-versicolor e Iris-virginica

\begin{tabular}{cccc}
\hline $\begin{array}{c}\text { Conjunto de } \\
\begin{array}{c}\text { Treinamento } \\
\text { (\%) }\end{array}\end{array}$ & $\begin{array}{c}\text { Acertos } \\
\text { (\%) }\end{array}$ & $\begin{array}{c}\text { Falsos } \\
\text { Versicolor } \\
\text { (\%) }\end{array}$ & $\begin{array}{c}\text { Falsos } \\
\text { Virginica } \\
\text { (\%) }\end{array}$ \\
\hline 50.00 & 64.00 & 26.00 & 10.00 \\
20.00 & 43.75 & 17.50 & 38.75 \\
\hline
\end{tabular}

Fonte: (Autor, 2020).

Tabela 6. Utilizando rede neural Perceptron, classificação para comprimento e largura da pétala das classes Iris-versicolor e Iris-virginica

\begin{tabular}{cccc}
\hline $\begin{array}{c}\text { Conjunto de } \\
\begin{array}{c}\text { Treinamento } \\
\text { (\%) }\end{array}\end{array}$ & $\begin{array}{c}\text { Acertos } \\
\text { (\%) }\end{array}$ & $\begin{array}{c}\text { Falsos } \\
\text { Versicolor } \\
\text { (\%) }\end{array}$ & $\begin{array}{c}\text { Falsos } \\
\text { Virginica } \\
\text { (\%) }\end{array}$ \\
\hline 60.00 & 97.50 & 0.00 & 2.50 \\
80.00 & 80.00 & 0.00 & 20.00 \\
\hline
\end{tabular}

Fonte: (Autor, 2020).
Figura 6. Pesos resultantes para a formação da reta: $w 0$ (bias) $=79, w 1=-52.40036, w 2=$ 69.20282 .

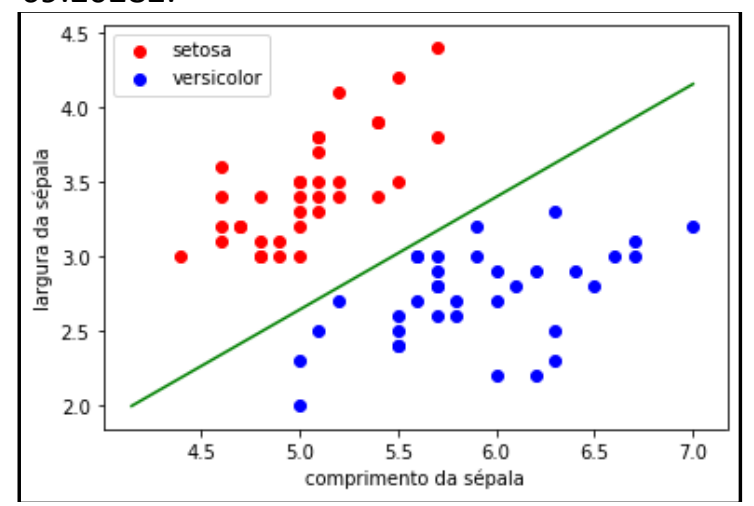

Fonte: (Autor, 2020).

A seguir a Figura 6 apresentando uma classificação com $100 \%$ de acertos da rede neural Perceptron, correspondendo a Tabela 1 e a Figura 7 apresentando uma classificação com o pior desempenho da rede neural Perceptron, com 43,75\% de acertos, correspondendo a Tabela 5.

Figura 7. Pesos resultantes para a formação da reta: $w 0$ (bias) $=148, w 1=25.499744, w 2=-$ 102.39855 .

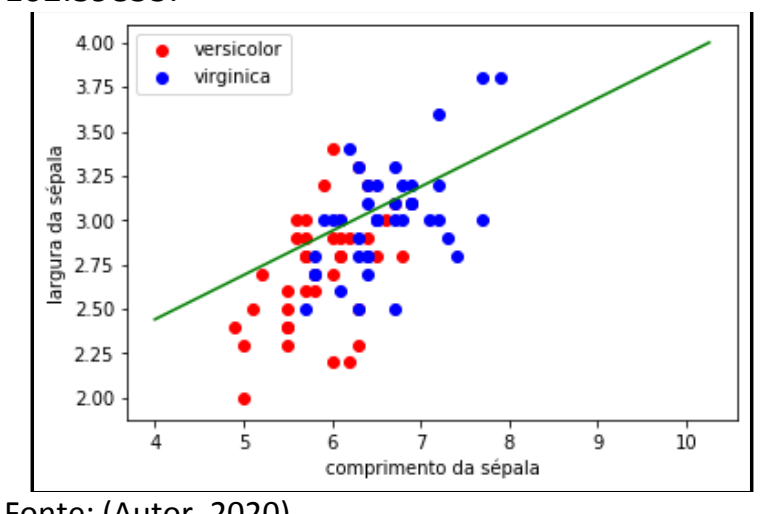

Fonte: (Autor, 2020).

\subsection{Adaline}

Tabela 7. Utilizando rede neural Adaline, classificação para comprimento e largura da sépala das classes Iris-setosa e Iris-versicolor

\begin{tabular}{cccc}
\hline $\begin{array}{c}\text { Conjunto de } \\
\text { Treinamento } \\
(\%)\end{array}$ & $\begin{array}{c}\text { Acertos } \\
\text { (\%) }\end{array}$ & $\begin{array}{c}\text { Falsos } \\
\text { Setosa (\%) }\end{array}$ & $\begin{array}{c}\text { Falsos } \\
\text { Versicolor } \\
\text { (\%) }\end{array}$ \\
\hline 30.00 & 100.00 & 0.00 & 0.00 \\
40.00 & 100.00 & 0.00 & 0.00 \\
80.00 & 100.00 & 0.00 & 0.00 \\
50.00 & 88.00 & 12.00 & 0.00 \\
\hline
\end{tabular}

Fonte: (Autor, 2020). 
Tabela 8. Utilizando rede neural Adaline, classificação para comprimento e largura da pétala das classes Iris-setosa e Iris-versicolor

\begin{tabular}{cccc}
\hline $\begin{array}{c}\text { Conjunto de } \\
\begin{array}{c}\text { Treinamento } \\
\text { (\%) }\end{array}\end{array}$ & $\begin{array}{c}\text { Acertos } \\
\text { (\%) }\end{array}$ & $\begin{array}{c}\text { Falsos } \\
\text { Setosa (\%) }\end{array}$ & $\begin{array}{c}\text { Falsos } \\
\text { Versicolor } \\
\text { (\%) }\end{array}$ \\
\hline 60.00 & 100.00 & 0.00 & 0.00 \\
70.00 & 100.00 & 0.00 & 0.00 \\
80.00 & 100.00 & 0.00 & 0.00 \\
50.00 & 98.00 & 2.00 & 0.00 \\
\hline
\end{tabular}

Fonte: (Autor, 2020).

Tabela 9. Utilizando rede neural Adaline, classificação para comprimento e largura da sépala das classes Iris-setosa e Iris-virginica

\begin{tabular}{cccc}
\hline $\begin{array}{c}\text { Conjunto de } \\
\text { Treinamento } \\
\text { (\%) }\end{array}$ & $\begin{array}{c}\text { Acertos } \\
\text { (\%) }\end{array}$ & $\begin{array}{c}\text { Falsos } \\
\text { Setosa (\%) }\end{array}$ & $\begin{array}{c}\text { Falsos } \\
\text { Virginica } \\
\text { (\%) }\end{array}$ \\
\hline 20.00 & 100.00 & 0.00 & 0.00 \\
30.00 & 100.00 & 0.00 & 0.00 \\
70.00 & 100.00 & 0.00 & 0.00 \\
80.00 & 90.00 & 10.00 & 0.00 \\
\hline
\end{tabular}

Fonte: (Autor, 2020).

Tabela 10. Utilizando rede neural Adaline, classificação para comprimento e largura da pétala das classes Iris-setosa e Iris-virginica

\begin{tabular}{cccc}
\hline $\begin{array}{c}\text { Conjunto de } \\
\text { Treinamento } \\
\text { (\%) }\end{array}$ & $\begin{array}{c}\text { Acertos } \\
\text { (\%) }\end{array}$ & $\begin{array}{c}\text { Falsos } \\
\text { Setosa (\%) }\end{array}$ & $\begin{array}{c}\text { Falsos } \\
\text { Virginica } \\
\text { (\%) }\end{array}$ \\
\hline 20.00 & 100.00 & 0.00 & 0.00 \\
30.00 & 100.00 & 0.00 & 0.00 \\
40.00 & 100.00 & 0.00 & 0.00 \\
50.00 & 100.00 & 0.00 & 0.00 \\
60.00 & 100.00 & 0.00 & 0.00 \\
70.00 & 100.00 & 0.00 & 0.00 \\
80.00 & 100.00 & 0.00 & 0.00 \\
\hline
\end{tabular}

Fonte: (Autor, 2020).

Tabela 11. Utilizando rede neural Adaline, classificação para comprimento e largura da sépala das classes Iris-versicolor e Iris-virginica

\begin{tabular}{cccc}
\hline $\begin{array}{c}\text { Conjunto de } \\
\begin{array}{c}\text { Treinamento } \\
\text { (\%) }\end{array}\end{array}$ & $\begin{array}{c}\text { Acertos } \\
\text { (\%) }\end{array}$ & $\begin{array}{c}\text { Falsos } \\
\text { Versicolor } \\
\text { (\%) }\end{array}$ & $\begin{array}{c}\text { Falsos } \\
\text { Virginica } \\
\text { (\%) }\end{array}$ \\
\hline 30.00 & 72.90 & 15.70 & 11.40 \\
20.00 & 46.25 & 11.25 & 42.50 \\
\hline
\end{tabular}

Fonte: (Autor, 2020).
Tabela 12. Utilizando rede neural Adaline, classificação para comprimento e largura da pétala das classes Iris-versicolor e Iris-virginica

\begin{tabular}{cccc}
\hline $\begin{array}{c}\text { Conjunto de } \\
\begin{array}{c}\text { Treinamento } \\
\text { (\%) }\end{array}\end{array}$ & $\begin{array}{c}\text { Acertos } \\
\text { (\%) }\end{array}$ & $\begin{array}{c}\text { Falsos } \\
\text { Versicolor } \\
\text { (\%) }\end{array}$ & $\begin{array}{c}\text { Falsos } \\
\text { Virginica } \\
\text { (\%) }\end{array}$ \\
\hline 40.00 & 96.70 & 1.70 & 1.70 \\
60.00 & 65.00 & 0.00 & 35.00 \\
\hline
\end{tabular}

Fonte: (Autor, 2020).

A Figura 8 apresenta uma classificação com $100 \%$ de acertos da rede neural Adaline, correspondendo a Tabela 7, e a Figura 9 apresenta uma classificação com 0 pior desempenho da rede neural Adaline, com 46.25\% de acertos, correspondendo a Tabela 11.

Figura 8. Pesos resultantes para a formação da reta: $w 0$ (bias) $=-0.9220933, w 1=-0.94848824$, $\mathrm{w} 2=1.3466693$.

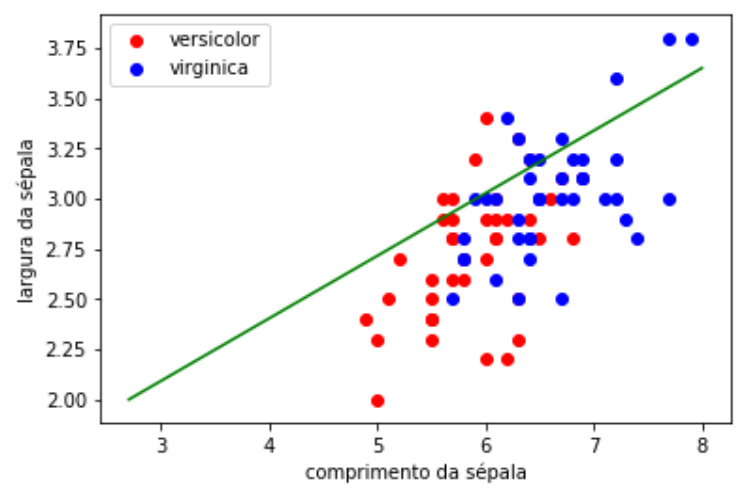

Fonte: (Autor, 2020)

Figura 9. Pesos resultantes para a formação da reta: $\mathrm{w0}$ (bias) $=-1.8686919, \mathrm{w} 1=0.5037405, \mathrm{w} 2$ $=-1.6156605$.

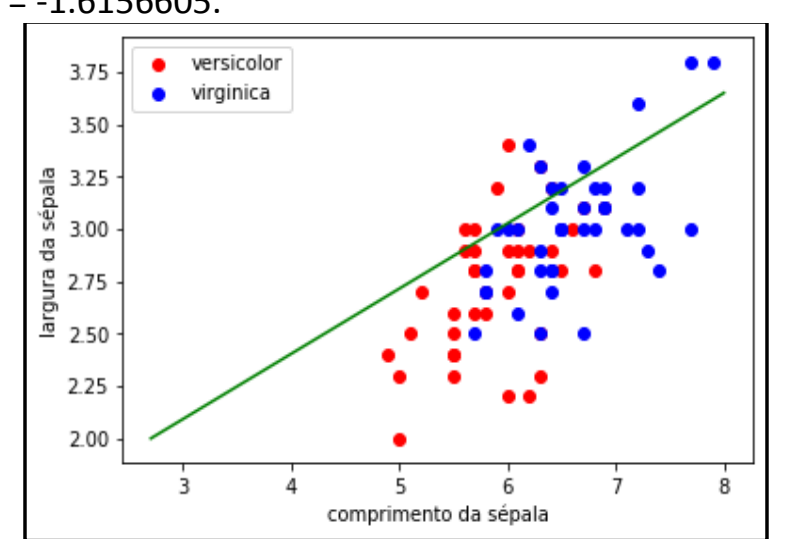

Fonte: (Autor, 2020)

\section{CONSIDERAÇÕES FINAIS}

Nas classificações para comprimento e largura da pétala observa-se nas Tabelas 2, 4, 8 e 10, que ambas redes neurais obtiveram $100 \%$ de acertos para o conjunto de 
treinamento utilizando $70 \%$ das amostras nas classificações entre as classes Iris-setosa e Irisversicolor e nas classificações entre Iris-setosa e Iris-virginica.

O melhor desempenho foi obtido pela rede neural Adaline nas classificações de comprimento e largura da pétala entre as classes Iris-setosa e Iris-virginica, visto na Tabela 10, onde apresentou $100 \%$ de acertos para todas os conjuntos de treinamento.

O Perceptron apresentou melhor desempenho nas classificações de comprimento e largura da pétala entre as classes Iris-setosa e Iris-virginica quando comparadas com as demais classificações, com $100 \%$ de acertos para dos conjuntos de treinamento utilizando $40 \%, 70 \%$ e $80 \%$ das amostras, vistos na Tabela 4 .

Os melhores desempenhos encontrados nas classificações entre as classes Iris-setosa e Iris-virginica ocorreram por conta dos pontos representando tais classes estarem mais afastados entre si do que os pontos representando Iris-setosa e Iris-versicolor ou Irisvirginica e Iris-versicolor. Pode-se visualizar tal fato comparando a posição dos pontos referentes as classes Iris-setosa e Iris-virginica com os pontos da classe Iris-versicolor na Figura 10.

Figura 10. Características das amostras do conjunto de dados no plano

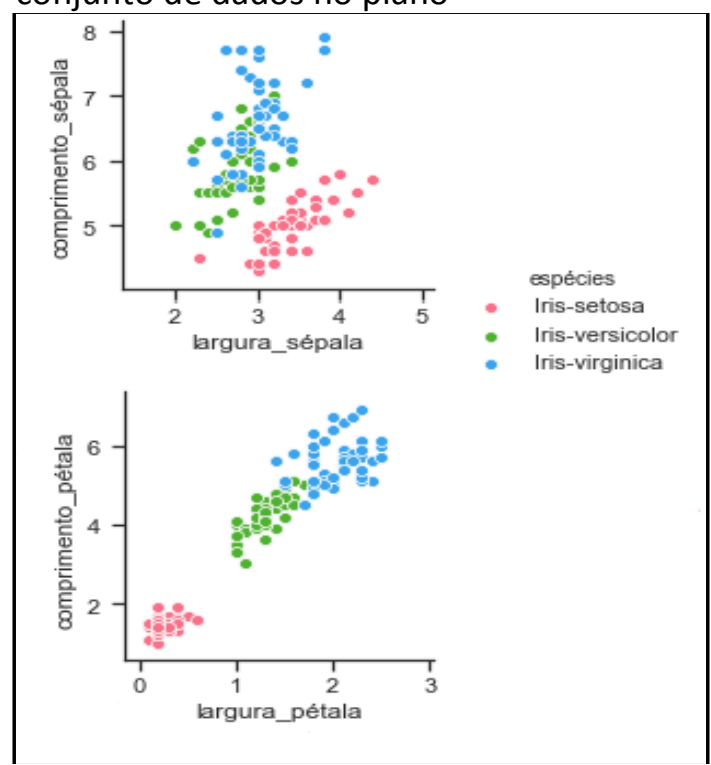

Fonte: (Autor, 2020).

A comparação entre os melhores desempenhos das redes neurais vistos nas Tabelas 4 e 10 mostra que o Adaline classifica melhor que o Perceptron, pois apresentou $100 \%$ de acertos para todas os conjuntos de treinamento enquanto o Perceptron apresentou $100 \%$ de acertos para 3 conjuntos de treinamento. A comparação entre as Tabelas $1 \mathrm{e}$ 7, também mostra um melhor desempenho do Adaline com 3 conjuntos de treinamento apresentando $100 \%$ de acertos enquanto o Perceptron possui apenas 2 conjuntos de treinamento apresentando $100 \%$ de acertos.

Ambas as redes neurais apresentaram o pior desempenho para a classificação de comprimento e largura da sépala entre Irisversicolor e Iris-virginica para o conjunto de treinamento utilizando $20 \%$ das amostras, vistos nas Tabelas 5 e 11, onde o Perceptron apresentou $43.75 \%$ de acertos e o Adaline $46.25 \%$.

O pior desempenho visto nas classificações entre as classes Iris-versicolor e Irisvirginica deve-se ao fato de que os pontos representando tais classes não podem ser linearmente separados principalmente para os valores de comprimento e largura da sépala, que podem ser visualizados no primeiro gráfico da Figura 10.

A comparação entre os piores desempenhos das redes neurais vistos nas Tabelas 5 e 11 mostra que o Perceptron classifica pior que o Adaline, onde o melhor desempenho apresentado pelo Perceptron em sua pior classificação é de apenas $64.00 \%$ de acertos enquanto o Adaline apresentou $72.90 \%$ de acertos.

Conclui-se que para o experimento realizado considerando como valor decisivo a quantidade de acertos totais entre as redes neurais, o Adaline teve melhor desempenho frente ao Perceptron. Sendo que o Adaline apresentou melhor desempenho na classificação para comprimento e largura da pétala entre as classes Iris-setosa e Iris-virginica dentre todas as demais classificações.

\section{REFERÊNCIAS}

Autor (2020). Redes Neurais. Disponível em: https://github.com/AllanaCampos/Redes-

Neurais. Acesso: 19 ago. 2020.

Data Science Academy (2019). Deep Learning Book. Disponível em: http://www.deeplearningbook.com.br/. Acesso: 02 jul. 2020.

Dua, D. e Graff, C. (2019). UCI Machine Learning Repository. Irvine, CA: University of California, School of Information and Computer Science. Disponível em: <http://archive.ics.uci.edu/ml>. Acesso: 02 jul. 2020. 
HUNTER, J.. et al. Matplotlib: Visualization with Python. 2020. Disponível em: https://matplotlib.org. Acesso: 02 jul. 2020.

MATOS, M. de. et al. Implementação de redes neurais perceptron e adaline em ambiente labview. Disponível em: http://repositorio.roca.utfpr.edu.br/jspui/bitstre am/1/10215/1/PG_COELE_2018_1_04.pdf.

Acesso: 02 jul. 2020.

MOREIRA, S. Rede Neural Perceptron Adaline. 2018. Disponível em: https://medium.com/ensina-ai/rede-neuralperceptron-adaline-8f69dc419d4e. Acesso: 17 Jul. 2020.

PYTHON (2020). Pandas. Disponível em: https://pypi.org/project/pandas/ Acesso em: 17 jul. 2020.

FISHER, R. A. The Use of Multiple Measurements in Taxonomic Problems. Annals of Eugenics, v. 7, n. 2, p. 179-188., 1936. Disponível em: http://www.comp.tmu.ac.jp/morbier/R/Fisher1936-Ann._Eugen.pdf. Acesso: 25 jul. 2020. https://doi.org/10.1111/i.1469-

1809.1936.tb02137.x

SHARMA, R. SIGgRAPH Now | Hands-on Workshop: Machine Learning and Neural Networks - Lecture 3. 2020. Disponível em: <https://www.youtube.com/watch?v=anASnKYI mSI\&t=1670s>. Acesso: 02 jul. 2020.

Silva, IN da, Danilo Hernane Spatti, e Rogério Andrade Flauzino (2010). Redes neurais artificiais para engenharia e ciências aplicadas. São Paulo: Artliber.

Waskom, Michael (2020). Seaborn. Disponível em: <https://seaborn.pydata.org>. Acesso: 17 jul. 2020. 